\title{
Mirar la historia de la arqueología uruguaya con lentes violetas
}

\section{Looking at the history of archaeology in Uruguay through violet lenses}

\author{
doi.org/10.48162/rev.46.005 \\ Eugenia Villarmarzo (D) orcid.org/0000-0002-2124-4798 \\ Jimena Blasco ${ }^{\ddagger}$ (D) orcid.org/0000-0002-1895-4473 \\ Elena Saccone* (D) orcid.org/0000-0002-1869-7294
}

\section{RESUMEN}

El artículo presenta los primeros resultados del proyecto "Mujeres en la arqueología. Desde las pioneras aficionadas hasta las profesionales de hoy". Esta línea de investigación surge como inquietud y necesidad de dar visibilidad y valorizar la producción teórica y académica de las mujeres en la construcción de la antropología y la arqueología en el Uruguay. La investigación en archivo y entrevistas busca recuperar la memoria de la participación de las mujeres en los estudios de arqueología de campo y laboratorio, y visibilizar el papel de las mujeres en la generación de los acervos y colecciones. El proyecto propone difundir y sensibilizar sobre el legado de las mujeres y su invisibilización dentro de la historización de la arqueología. Se identifica que diversas mujeres, tanto uruguayas como extranjeras, jugaron un papel de gran relevancia para el desarrollo de la disciplina, así como para la creación de las grandes colecciones de los museos nacionales.

Palabras clave: arqueología de género, antropología feminista, historia de la arqueología, arqueología Uruguay

\section{ABSTRACT}

$\phi$ Laboratorio de Arqueología del Paisaje y Patrimonio (LAPPU), Facultad de Humanidades y Ciencias de la Educación, Unidad Asociada al CURE, Universidad de la República. eugenia.villarmarzo@lappu.edu.uy \$ Laboratorio de Arqueología del Paisaje y Patrimonio (LAPPU), Facultad de Humanidades y Ciencias de la Educación, Unidad Asociada al CURE, Universidad de la República. jimena.blasco@lappu.edu.uy

* Laboratorio de Arqueología del Paisaje y Patrimonio (LAPPU), Facultad de Humanidades y Ciencias de la Educación, Unidad Asociada al CURE, Universidad de la República. elena.saccone@lappu.edu.uy 
This paper presents the first results of the research project "Women in archaeology: From amateur pioneers to today's professionals." This line of research emerged as a concern and a need to make visible and revalue the theoretical and academic production of women in the development of anthropology and archaeology in Uruguay. Through archival research and interviews, we seek to recover the memory of women participating in field archaeology and laboratory activities, while at the same time, making visible the role of women in the production of museum collections. The project aims at disseminating and raising awareness about the legacy of women and their invisiblization in the history of archaeology. We identify several women from Uruguay and other countries who played a significant role in the development of the discipline and in the creation of the collections at national museums.

Keywords: gender archaeology, feminist anthropology, history of archeology, uruguayan archaeology

Recibido: 24/04/2021

Aceptado: 06/10/2021

\section{INTRODUCCIÓN}

Como escribió Virginia Wolf, "para la mayor parte de la Historia, ‘Anónimo' era una mujer.” Wolf (2008 [1929]). En este trabajo presentamos los primeros avances de un proyecto que partió del interés inicial por conocer el trabajo de las mujeres que han contribuido al desarrollo de la arqueología en Uruguay. El proyecto "Mujeres en la arqueología. Desde las pioneras aficionadas hasta las profesionales de hoy" nació el 5 de febrero de 2019 a partir de la consulta de una colega sobre alguna exploradora o antropóloga pionera de Uruguay. A partir de esta pregunta, comenzamos a intercambiar los escasos datos que teníamos. Sin embargo, decidimos que un día, no muy lejano, íbamos a comenzar a contar las historias de las pioneras de la arqueología uruguaya. Debido a nuestras múltiples ocupaciones y responsabilidades, la investigación sistemática quedó relegada y nos limitamos a la recopilación de información que buscábamos en nuestros tiempos libres, o que llegaba a nuestras manos de manera fortuita. Este fue el germen que permaneció latente durante varios meses, esperando el momento propicio para desarrollarse.

En 2020, la pandemia de COVID-19 resultó crucial para retomar esta iniciativa que había quedado en algo muy incipiente. Los meses de confinamiento, las tareas de cuidados y la agudización de las restricciones para las arqueólogas en el ámbito académico y laboral, volvieron a hacernos reflexionar sobre el papel de las mujeres en nuestra sociedad en general y en la arqueología en particular, sobre las desigualdades, las cargas invisibles, la falta de reconocimiento, entre otros temas. Esto nos llevó a retomar la idea del proyecto y buscar, de alguna manera, en 
una especie de solidaridad intergeneracional, hacer justicia social y reivindicar el trabajo de aquellas mujeres que, con escaso reconocimiento hasta hoy, fueron parte del quehacer arqueológico en nuestro país desde sus inicios a principios del S. XX.

De este modo, el objetivo general del proyecto es dar visibilidad al papel de las mujeres en la antropología y la arqueología uruguayas. En particular nos interesa valorizar su producción teórica y académica en temas de prehistoria y arqueología, y recuperar la participación de las pioneras en trabajos de campo. Utilizamos el término "lentes violetas", o también "lentes de género", que es una expresión del feminismo que refiere al desarrollo de una actitud crítica-reflexiva frente a situaciones que en otras condiciones de análisis veríamos naturales, para detectar violencias y sexismo. A la par, también buscamos reflexionar sobre el rol de las mujeres en la generación, conservación y socialización de las colecciones arqueológicas que hoy componen los museos del país. La investigación pretende, al mismo tiempo, rescatar la visión de estas pioneras sobre la prehistoria y la construcción de la arqueología nacional. El fin último es dar a conocer, difundir y sensibilizar sus aportes, poniendo sobre la mesa la discusión sobre la construcción histórica de la arqueología como una disciplina androcéntrica (Gero, 1999), gestada dentro de una sociedad patriarcal. El androcentrismo es lo que ha dominado la visión sobre las sociedades del pasado, sobre los roles y sobre la historia de la disciplina (con sus consecuentes implicancias teóricas, epistemológicas y metodológicas).

\section{Las arqueologías feministas}

Existen distintos ejes o categorías (siguiendo a Wylie, 1997) desde los cuales se puede trabajar en arqueología de género y arqueología feminista. Una de ellas sería la de los contenidos, es decir, la forma en que se interpreta y se representa o se invisibiliza el rol de las mujeres en el pasado -tanto en las producciones académicas como desde los museos y las propuestas educativas y de divulgación (entre muchos otros: Adovasio et al., 2007; Colomer et al., 1999; Pacheco, 2019; Prados Torreira y López Ruiz, 2017). La segunda tiene que ver con la inequidad, es decir, las formas en que las arqueólogas han sido marginalizadas en el ámbito académico y profesional. Según Wylie (1997) estos análisis feministas constituyen los trabajos empíricamente más ricos de este tipo, que documentan no solo patrones persistentes de apoyo diferencial, de capacitación y avance para las mujeres en arqueología, sino también patrones arraigados de segregación de género en las áreas en las que las mujeres suelen trabajar (Wylie, 1997: 83). Para nosotras, a una tercera categoría pertenecen estudios más recientes sobre acoso y violencias de género (hacia las mujeres y las disidencias) (Colegio de Arqueólogas y 
Arqueólogos de Chile [CAARCH], s.f.; Tavera Medina, 2019; Voss, 2021 a, b, entre otros).

Concebimos este primer trabajo como una etapa inicial en el camino a una arqueología feminista. Si bien la metodología y las líneas de trabajo abarcan un mundo más amplio, nos circunscribimos a la segunda categoría, en la que intentaremos poner sobre la mesa el nombre, las producciones y los logros de estas mujeres que nos precedieron. Es necesario aclarar que, por cuestiones de espacio y tiempo, hicimos un recorte y presentamos la información referente a los primeros años que aborda la investigación, que incluye un panorama muy breve sobre las primeras egresadas.

Como puntapié inicial -algo que sería obvio si estuviéramos hablando de varones- resaltaremos las figuras y los éxitos. Por supuesto que estas carreras "de éxito" han estado atravesadas por fracasos, imposiciones, violencias y ciertamente también de negaciones. De la misma manera, nuestro propio trabajo comienza a ser mirado con recelo, y hay resistencias y reacciones que, por las razones anteriormente esgrimidas, serán desarrolladas en ulteriores trabajos. En este sentido, entendemos que la arqueología de género amplía los marcos interpretativos al considerar el género en el estudio del pasado como categoría estructural. Pero no toda arqueología de género es arqueología feminista ya que la segunda es una acción y opción política. Estas arqueologías, según Montón Subías (2014), van a aportar una dimensión humanista y el compromiso político con el fin del patriarcado. Según la autora, "promueven un cambio de cultura disciplinaria que acabe con sus sesgos sexistas y heterosexistas" (Montón Subías, 2014: 244).

El género como categoría analítica empezó a utilizarse en la década de 1980 y dio comienzo a una etapa de introspección con distintos alcances (Conkey y Spector, 1984). En esa época se realizaron reuniones bajo esa temática principalmente en Europa y Estados Unidos. Desconocemos hasta qué punto estas discusiones del norte tuvieron influencia en las arqueologías latinoamericanas y hasta donde sabemos, a excepción de la introducción al libro Género y Etnicidad en la Arqueología Sudamericana desarrollada por Benjamín Alberti y Verónica Williams (2005), no existe aún una síntesis del desarrollo de la arqueología feminista en este continente. Sería interesante ver de qué modo estos movimientos incidieron en nuestras arqueologías. Según el análisis realizado por Rodrigo Navarrete (2010), el reconocimiento del enfoque feminista o de género es reciente en el contexto latinoamericano, en especial en Argentina, México y Venezuela. Por un lado, según parece al autor, la academia latinoamericana no está aún dispuesta a otorgarle el lugar central que deben tener los análisis de género tanto sobre el pasado como con la producción de su conocimiento. No obstante, en nuestras latitudes existe una academia con una fuerte tradición crítica epistemológica y 
política, como por ejemplo, la Arqueología Social que, desde los setenta, y como escuela de pensamiento marxista genuinamente latinoamericana, creó un nicho para el análisis de género en un contexto en el que el análisis de clases y la comprensión del discurso arqueológico como producto cultural y político ya eran comunes para nuestra comunidad (Navarrete, 2010).

En el caso uruguayo no hay un desarrollo teórico y metodológico de la arqueología que incluya al género como categoría estructural, aunque algunas tesis de grado han abordado cuestiones de género (Brum, 2008). A pesar de la proximidad, esto no fue así con la antropología social, donde autoras como Susana Rostagnol (1989) empezaron a reflexionar en torno a la categoría de género y poder ya desde la década de 1980 .

\section{La historia de la arqueología en el Uruguay}

Como veremos en este apartado, en Uruguay varios investigadores -en su amplia mayoría varones- se han encargado de desarrollar tanto una cronología de la arqueología nacional, como una historia del desarrollo del pensamiento teórico con el mismo alcance. Sin embargo, consideramos que no se ha prestado especial atención aún a la visión y aportes de las arqueólogas uruguayas a la disciplina. En este apartado buscamos dar un marco general para plasmar los resultados que se desarrollarán más adelante en este trabajo, y comenzar a introducirnos en la problematización de esta invisibilización.

La principal referencia en la reconstrucción de la historia de la arqueología en el Uruguay es el trabajo monográfico de Leonel Cabrera Pérez (1988) (retomado luego en Cabrera Pérez y Curbelo, 1992; Cabrera Pérez, 2004, 2011, entre otros). El autor refiere inicialmente a una periodización en tres etapas. La primera comienza a finales del siglo XIX y continúa por gran parte de la primera mitad del siglo XX. Estos inicios se vinculan a investigadores como José H. Figueiras, Benjamín Sierra y Sierra y Orestes Araújo. Sus trabajos iniciales se caracterizaron, según el autor, por una "escasa profundidad temporal", hallazgos fortuitos y "enfoques simplistas" (Cabrera Pérez, 1988: 10). En ese entonces se comenzaron a formar las primeras grandes colecciones de materiales arqueológicos y estos se relacionaron con las etnias etnohistóricas (Cabrera Pérez, 1988). Dentro de esta primera etapa se funda, en 1926, la Sociedad de Amigos de la Arqueología (ver más abajo). La segunda etapa está marcada por nuevas inquietudes y comienza, según Cabrera Pérez (1988), con la fundación de la Asociación de Lingüística y Antropología de Montevideo en 1948, que luego se convertiría en 1951 en la Sociedad de Antropología del Uruguay. Para finalizar esta segunda etapa, en 1969 se crea el Centro de Estudios Arqueológicos (CEA) cuyo principal objetivo era el 
"estudio científico de la Arqueología Americana y especialmente de la Prehistoria del Uruguay" (CEA, 1989), pero además promovía la difusión de los conocimientos producidos, la realización de encuentros internacionales, la enseñanza y la cooperación con diversas instituciones. La tercera etapa se inicia a mediados de la década de 1970 -en pleno gobierno de facto- con la creación de la Licenciatura en Ciencias Antropológicas en la Facultad de Humanidades y Ciencias de la Universidad de la República y la Misión de Rescate Arqueológico de Salto Grande (en adelante MRASG). La primera generación de arqueólogos y arqueólogas profesionales ingresan en 1976 a cursar sus estudios universitarios y comienzan a formarse con especialistas extranjeros. La dirección de la carrera fue encomendada al argentino Antonio Austral, quien desarrolló excavaciones en nuestro país. Estas excavaciones y las de la MRASG, fueron las primeras experiencias de campo y laboratorio para los y las primeros/as estudiantes. Aunado a esto, la Misión de Salto Grande dio visibilidad internacional a la arqueología uruguaya, ya que participaron equipos de investigadores e investigadoras de diversas nacionalidades (Cabrera Pérez, 1988).

Luego de estos tres momentos definidos en el devenir de la arqueología uruguaya, Cabrera Pérez (1988) realiza una actualización de su trabajo en la que añade una nueva etapa que corresponde a los trabajos de "los primeros arqueólogos uruguayos" (Cabrera Pérez, 2011: 110) egresados de la licenciatura y a los primeros grandes proyectos en el país en el contexto de una arqueología de rescate vinculados a diversas obras. Este periodo había sido definido por Antonio Lezama como "la crisis de los 80 " o "suerte de parálisis en la arqueología nacional" en tanto que insertos en una lamentable situación dada por la dictadura militar, la intervención de la universidad, el exilio y "el forzoso 'encasillamiento' ideológico" de quienes se quedaron en el país, el encuadramiento de "los jóvenes" en la cátedra universitaria creada, así como el cerramiento del campo de investigación a los aficionados (Lezama Astigarraga, 1995: 4). Esto último, según el autor, sería consecuencia de la promulgación de la Ley de Patrimonio (Ley $\mathrm{N}^{\circ}$ 14.040/71). Además, agrega: "podríamos hablar de una barrera psicológica ante la presencia de arqueólogos profesionales actuando en nuestro territorio que inhibía la acción de los arqueólogos nacionales esencialmente autodidactas." (Lezama Astigarraga, 1995: 4). En esta misma línea, más tarde Carmen Curbelo reflexionará:

"Los primeros egresados de la academia se proponen como objetivo marcar claramente el quiebre de pensamiento teórico y de conceptualización, hasta en lo semántico diríamos, del término arqueología, elaborando un discurso de legitimación [...] Este discurso implicó la necesidad de definir la arqueología como una disciplina científica con mayúsculas para separar su concepción de las 
prácticas de coleccionismo entendido como un amateurismo que carecía de falta de rigor científico..." (Curbelo, 2004:272).

En este sentido, la autora hizo una distinción entre conocimiento científico y conocimiento autoritario (Curbelo, 2004). Aunque se basó en Cabrera Pérez (1988), evita hablar de etapas o momentos para la historia de la teoría arqueológica uruguaya y se refiere a "impulsos", "puntos de inicio" o "focos" (Curbelo, 2004: 265). Frente a esto, señala que los movimientos de producción de conocimiento en la teoría arqueológica uruguaya se superponen unos a otros: "Muchas veces una línea de investigación o una postura teórica responden a un interés de oponer unas ideas con otras generadas anterior o paralelamente" (Curbelo, 2004: 265). En la misma línea que Cabrera Pérez (2011), la autora hablará de "cuarto foco o gran quiebre" para referirse a "El surgimiento de la arqueología académica uruguaya" (Curbelo, 2004: 265). Sin embargo, su presentación del CEA dista de la de “entusiastas” presentada por Cabrera Pérez (2011: 94).

"Los trabajos del C.E.A. marcan las primeras investigaciones arqueológicas en territorio nacional teniendo en cuenta la aplicación consciente de todos los pasos que involucra una investigación [...] se podrá discrepar pero en este caso no existen inconsistencias entre los objetivos planteados y la ejecución y los resultados exhibidos" (Curbelo, 2004: 270).

En relación con esto, al hablar sobre los marcos teóricos y criterios dominantes en las tipologías líticas uruguayas, Cabrera Pérez (2004) argumenta que con la profesionalización de la arqueología comienzan a atenuarse los sesgos propios de los criterios tipológicos importados de Europa que imperaron hasta la década de 1980 -más allá de que hasta el momento seguía existiendo una predilección por el estudio de los artefactos líticos tallados en detrimento de los modificados mediante pulido, picoteado y/o abrasión y de la cerámica-. A excepción de la mención a la arqueóloga Niède Guidón -responsable científica del Rescate Arqueológico de Salto Grande-, es importante notar cómo la historia que describe está absolutamente dominada por una producción teórica altamente masculinizada, algo común para los períodos de la arqueología uruguaya a los que Cabrera Pérez hace referencia.

En este sentido, José M. López Mazz (2004) desarrolla un análisis teórico sobre el rol de la arqueología en la construcción del pasado. Hace un repaso por la historia de la disciplina y realiza una exposición argumentativa para indicar cómo, debido a los modelos explicativos de inspiración difusionista imperantes hasta la década de 1980, la arqueología consideró marginal al desarrollo cultural prehispánico -especialmente en las tierras bajas de Uruguay-, y fue funcional a un 
discurso oficial que negó a las minorías étnicas en la construcción de la identidad (López Mazz, 2004). Asimismo, pone sobre la mesa las tensiones ideológicas y disciplinares generadas entre arqueólogos, antropólogos, historiadores, periodistas y políticos en relación con la producción de esta visión hegemónica. Nuevamente, vemos cómo los protagonistas de estas acaloradas discusiones son varones pertenecientes a una élite de intelectuales de la época: Renzo Pi Hugarte, Daniel Vidart, José de Torre Wilson, Julio María Sanguinetti, Amir Ahmed, Gustavo Verdesio, Carlos Maggi, Danilo Antón, el propio autor, entre otros (López Mazz, 2004). De esta forma entendemos que queda en evidencia un predominio de la voz masculina en la construcción del relato oficial de la Historia. A propósito del rol de la arqueología como productora de un conocimiento que puede aportar a la sociedad herramientas para entender el mundo y transformarlo, el autor propone lo siguiente:

"Resulta fundamental el renovado vínculo entre educación y arqueología para la actualización de las identidades minoritarias, que a pesar de no tener siempre los documentos jurídicos más adecuados a su causa, pueden contar con la cultura material. Una cultura material, que resiste el manejo ideológico, y que al expresar la diversidad cultural permite forjar identidades desalineadas, fuera de las relaciones sociales de tipo patriarcal, y de la sesgada herencia colonial." (López Mazz, 2004: 199, destacado por las autoras).

Si bien concordamos con el autor en que es posible y necesario considerar a la cultura material como soporte para la construcción de discursos alternativos al hegemónico (patriarcal y colonialista), nos preguntamos cómo esta puede ser capaz de resistir al manejo ideológico, cuando los métodos arqueológicos empleados para recuperar y clasificar ese registro responden a intereses y preguntas de investigación guiados por visiones androcéntricas sobre las sociedades del pasado y del presente-; o cuando la dinámica y el contexto de investigación están dominados por la reproducción de relaciones laborales machistas.

\section{METODOLOGÍA}

La investigación se circunscribe temporalmente desde principios del $\mathrm{S}$. XX, específicamente desde la fundación de la Sociedad de Amigos de la Arqueología (en adelante SAA) hasta el año de los primeros egresos de la carrera profesional en arqueología de la Licenciatura de Ciencias Antropológicas de la Facultad de Humanidades y Ciencias (1983-1984). Para el análisis se utilizan fuentes documentales, así como entrevistas a mujeres que fueron y son parte de esta historia que queremos reconstruir. 
Las fuentes documentales abarcan revistas, que incluyen actas de reuniones de las sociedades científicas, boletines, anales y actas de encuentros y congresos, artículos de prensa, bibliografía, archivos personales y el archivo digital con registros genealógicos en línea de la Iglesia de Jesucristo de los Santos de los Últimos Días, Familysearch.org. Realizamos también relevamiento de archivo fotográfico, con registros de salidas de campo, asistencia a congresos, etc. En numerosas fotografías aparecen más mujeres de las que se relevan en las publicaciones.

Las entrevistas realizadas estuvieron enfocadas en las mujeres pertenecientes al CEA y en las egresadas de las primeras generaciones de la Licenciatura en Ciencias Antropológicas. Las entrevistas realizadas son semidirigidas a través de una pauta previamente diseñada que tiene cuatro ejes temáticos: 1) motivación e inicio de la labor arqueológica; 2) labor arqueológica y mujeres en la arqueología; 3) otras mujeres arqueólogas (compañeras y referentes) y 4) reflexiones finales respecto de mujeres y arqueología. La entrevista inicia con un consentimiento informado oral en el que se solicita permiso para grabar la charla, utilización de los datos, anonimato, uso de la imagen. Debido a las restricciones de movilidad y cuidados de las personas mayores en el contexto de COVID-19, la mayor parte de las entrevistas fueron realizadas de forma virtual.

Para el registro y sistematización de la información, realizamos el diseño de fichas biográficas de mujeres que contienen la siguiente información: nacimiento y fallecimiento (ciudad y fecha), estudios, actividades de investigación realizadas en Uruguay, otros datos biográficos de interés, publicaciones de la autora, publicaciones sobre la autora, investigaciones, páginas web, entradas en wikipedia. Las fichas se van completando a medida que avanza la investigación y a través de entrevistas.

\section{AVANCES Y PRIMEROS RESULTADOS}

Los avances de la investigación permiten entrever cómo el papel que jugaron diversas mujeres -tanto uruguayas como extranjeras- en el desarrollo de la arqueología nacional, a pesar de haber sido sistemáticamente opacado por las corrientes dominantes, fue de gran relevancia en diversas áreas de la disciplina, así como en la creación, conservación y socialización de las grandes colecciones de los museos nacionales.

Hasta el momento, hemos realizado ocho entrevistas repartidas entre aquellas mujeres que participaron del CEA y las primeras egresadas de la licenciatura en Antropología, y hemos contactado a los familiares de quienes han fallecido. Asimismo, contamos con 28 fichas de mujeres que participaron de los 
inicios de la arqueología nacional. Los datos relevados en estas 28 fichas se presentan a continuación en cuatro secciones, que abordan, intentando respetar un orden cronológico a: 1) las pioneras; 2) las mujeres de la Sociedad de Amigos de la Arqueología; 3) las mujeres integrantes del Centro de Estudios Arqueológicos y 4) un avance breve de las indagaciones sobre las primeras egresadas de la licenciatura en Ciencias Antropológicas, con orientación en Prehistoria y Arqueología, de la Facultad de Humanidades y Ciencias (Universidad de la República).

\section{Las pioneras}

Leila Carmen Tuya Martínez, creadora/fundadora del Museo Arqueológico del Cordón

Leila Carmen Tuya Martínez nació en Mercedes el 3 de octubre de 1909 y murió en Montevideo el 15 de octubre de 1996. Estudió magisterio en Montevideo y trabajó como maestra en la escuela $\mathrm{N}^{\circ} 4$ de Mercedes hasta que, en el año 1942, contrajo matrimonio con Carlos Maeso Tognochi (1892-1963), se radicó en Montevideo y abandonó la docencia (Arruabarrena y Vallvé, 2009).

Además de realizar actividades de campo junto a su esposo (Figura 1) y de acompañarlo en sus viajes y conferencias, desarrolló estudios sobre los materiales cerámicos de la colección, dio conferencias y publicó trabajos de su autoría. Luego de la muerte de su esposo, Leila creó la Fundación Carlos Maeso Tognochi y reformó su casa para montar el Museo Arqueológico del Cordón (MEC, 2017; Suárez, 2000). Se encargó tanto del montaje de la exposición como de la sistematización de la información, la conservación, registro e inventario de las piezas de la colección. El museo permaneció abierto durante treinta años, y recibió las visitas del público general, grupos de escolares, de estudiantes de secundaria y estudiantes de grado de la carrera de Arqueología que realizaron sus prácticas con la colección (MEC, 2017).

En 1972 presentó en el Primer Congreso Nacional de Arqueología de Uruguay (ciudad de Fray Bentos) un Informe sobre el material cerámico estudiado por el arqueólogo Carlos Maeso (Tuya de Maeso, 1973). Además, dio notas en revistas y editó y publicó el libro Investigaciones arqueológicas (1977), en el cual se compilan los trabajos, inéditos en su mayoría, de Carlos Maeso, en los que el investigador hace constante referencia a la compañía de su esposa:

"Una hermosa mañana del mes de abril de 1959, salimos con mi señora, en bote, de Villa Soriano para el Campo Morgan y desde aquí nos dirigimos al 
arroyo Caracoles a pie. Encontramos diseminados en una barranca fragmentos de cerámica, labrados, finos y gruesos, pocos fragmentos de urnas. Material de hueso, la mayoría de las piezas destrozadas. Una pieza original de hueso, desgastada, que considero una punta de lanza con manguito para enastar" (Maeso Tognochi, 1977: 97, cursiva de las autoras).

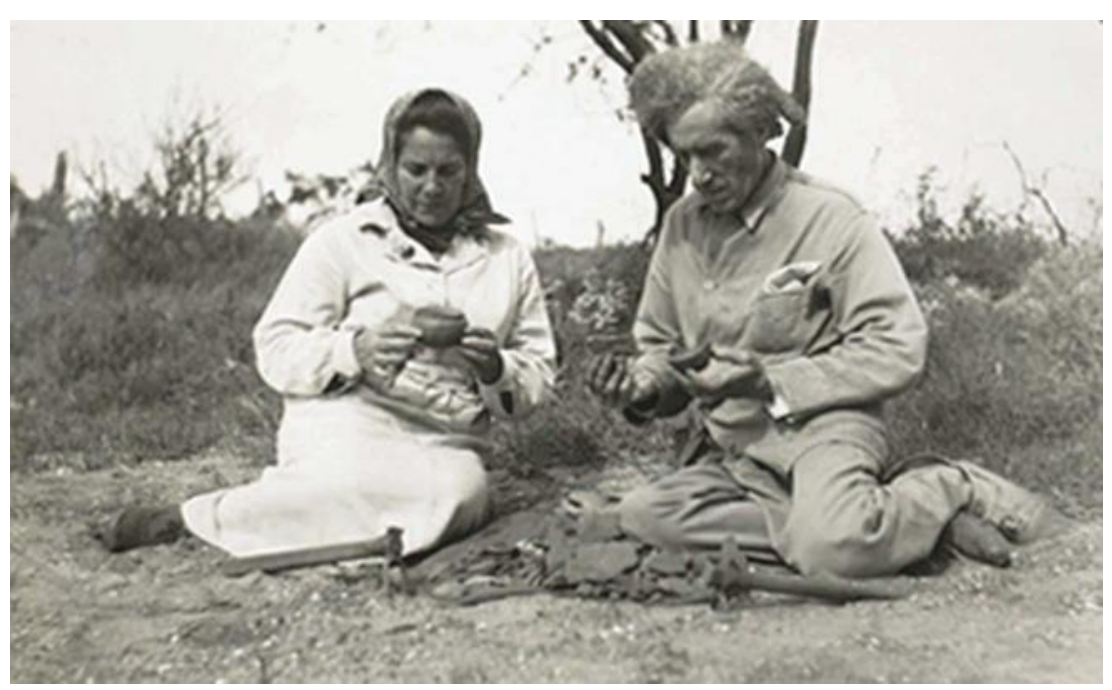

Figura 1. Leila Tuya y Carlos Maeso Tognochi. Imagen recuperada de: Exposición arqueológica | Ministerio de Educación y Cultura (www.gub.uy)

Si bien es innegable el reconocimiento de Carlos Maeso a la labor de su esposa en el trabajo de campo realizado, el autor hace un cambio gramatical en el pronombre al referirse a las interpretaciones: de "encontramos" a "considero". Sin embargo, en 1980 Leila publica un libro de su autoría titulado Cerámica indígena de la República Oriental del Uruguay. Consideraciones sobre la cerámica de yacimientos arqueológicos investigados en los departamentos de Colonia, Soriano y Río Negro. Se trata del análisis de materiales cerámicos de la colección, el cual está acompañado de dibujos realizados por la autora (Figura 2).

"Es impositivo el deber de realizar una breve reseña sobre la cerámica sistemáticamente conseguida por mi esposo, el arqueólogo Carlos Maeso Tognochi, y por mí, en las estaciones indígenas de La Blanqueada, Rincón de la Higuera, Islas del Naranhjo e Infante, departamento de Soriano; Campo Morgan, arroyo Caracoles e Isla del Vizcaino, departamento de Río Negro.

Las excavaciones que se efectuaron siguiendo un plan estratigráfico dieron como resultado el relevamiento de gran cantidad de fragmentos de 
cerámica, lisos y labrados, finos y gruesos; vasijas enteras, otras fragmentadas que fueron reconstruidas" (Tuya de Maeso, 1980: 9).
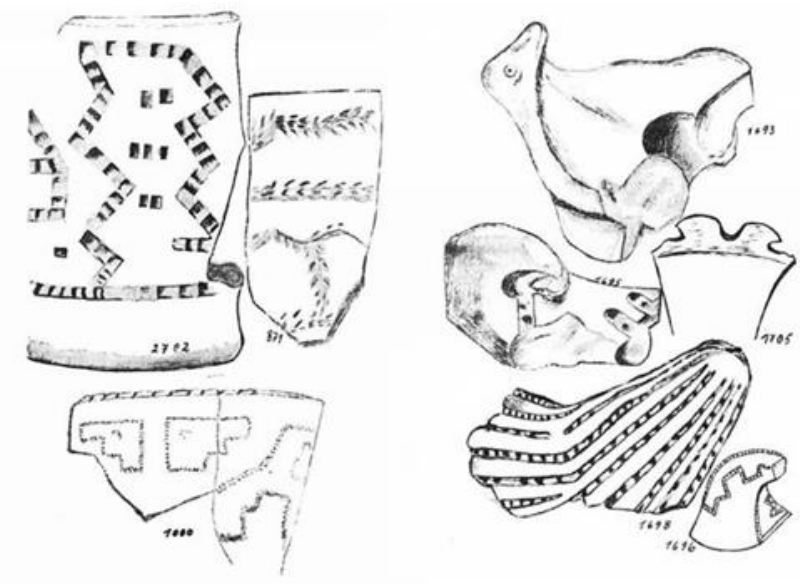

Figura 2. Dibujos de bordes de cerámica decorados (izquierda) y de apéndices de alfarería "gruesa" (derecha) realizados por Leila Tuya (Extraídos de Tuya de Maeso, 1980: 36, 89).

En 1996, año del fallecimiento de Leila, la colección fue declarada Monumento Histórico Nacional bajo el nombre de "Colección Carlos Maeso Tognochi" por medio de la resolución $\mathrm{N}^{\circ}$ 1019/996 (ROU, 1996), invisibilizando por completo su labor en la conformación e investigación de la colección, así como en su documentación, conservación y socialización a través de la difusión en escuelas y de la musealización. A partir de este momento, la Comisión de Patrimonio Cultural de la Nación (CPCN) se hizo cargo de la tutela y procedió a su inventario, acondicionamiento y conservación. En el año 2008, el Ministerio de Educación y Cultura (MEC), a través de la CPCN, adquirió la colección. Un año más tarde, se inauguró el edificio donde se emplazaría el Museo Maeso en Villa Santo Domingo de Soriano (Departamento de Soriano, lugar de nacimiento de Leila) con una exposición temporal de paneles con información titulada "Carlos Maeso y Leila Tuya. Pioneros de la arqueología uruguaya" (Arruabarrena y Vallvé, 2009). Es importante señalar que, además de reconocer a Leila como pionera, se destaca su figura a través de una breve biografía igual que la de Carlos Maeso (Arruabarrena y Vallvé, 2009). Si bien en 2017 se inauguró la exposición permanente "Arcilla, piedra y hueso a la orilla del Hum" (MEC, 2017) con una selección de piezas, la colección aún permanece almacenada en el Museo Nacional de Antropología, y espera pasar a custodia de la Intendencia de Soriano.

Cabe señalar, además, que en el año 2011 la Junta Departamental de Soriano aprobó un nuevo Nomenclátor para la ciudad de Mercedes, en cuyo listado 
se designó a la calle $\mathrm{N}^{\circ} 60$ con el nombre de "Mtra. Leila Tuya". A propósito, el arqueólogo Aparicio Arcaus ${ }^{1}$ nos cuenta lo siguiente:

"Leila es muy querida acá en Mercedes [...] Acá en Mercedes a Leila la conocían en particular antes de estar con Maeso, eh... ya era Leila sobre todo porque era maestra de escuela [...] Entonces la conoce muchísima gente de su tarea docente [...] Y por eso no solo fue la esposa de Maeso sino que tuvo, justamente, una identidad propia ligada a su tarea docente y a quién era ella" (Com. pers. Aparicio Arcaus, abril de 2021).

\section{Bell Clavelli, primera Directora del Museo Nacional de Antropología}

Bell Clavelli Gamberoni nació en Uruguay, el 10 de noviembre de 1918 y falleció en 1990 (Figura 3). Maestra de profesión, estudió y tomó clases de dibujo y pintura con Guillermo Laborde en la Escuela Industrial, junto con sus amigas y colegas Mercedes “Coca” Antelo y Quela Rovira (Bandrymer y Pontet, 2006). Estas mujeres fueron pioneras en la introducción del arte en el aula escolar, lo cual generó como resultado una exposición de dibujos hechos por niños y niñas de escuelas públicas de Montevideo que tuvo lugar en el Subte Municipal en el año 1947, y que recibió numerosos elogios por la élite cultural de la época (Bandrymer y Pontet, 2006). En esta iniciativa se vio involucrado un grupo de maestras interesadas en la arqueología y las culturas precolombinas de América, aspecto que las motivó a viajar por el continente e incluso participar en una excavación arqueológica en Paracas en la década de 1940 (Bandrymer y Pontet, 2006). Este es el caso de las maestras y artistas plásticas Quela Rovira, también alumna del Taller Torres García, e Isabel Gandola, comisionada por el Consejo Departamental de Montevideo para adquirir piezas arqueológicas de México, Guatemala, Costa Rica y Perú con el objetivo de crear el Museo Municipal de Arte Precolombino (Museo de Historia del Arte [MuHAr], s.f.).

Bell Clavelli se casó con Francisco Olvieras Acosta (1896-1987), profesor de Ciencias Naturales del Instituto de Magisterio y fundador del Centro de Estudios de Ciencias Naturales (Museo Nacional de Historia Natural [MNHN], s.f.), el cual compartía miembros con la Sociedad de Amigos de la Arqueología y el Centro de Estudios Arqueológicos (Bandrymer y Pontet, 2006). Oliveras fue quien generó, junto con la colaboración de su esposa y de varios colegas -entre quienes destacan numerosas maestras tales como Mercedes Antelo-, una de las colecciones arqueológicas más grandes de nuestro país, con un total de 102.432 piezas, a las que se les suman cerca de 80.000 objetos más que fueron clasificados como 
paleontológicos, zoológicos y geológicos (Museo Nacional de Antropología [MNA], s.f. a; MNHN, s.f.).

Cabe destacar que Bell Clavelli y Mercedes Antello fueron ilustradoras de la revista escolar "El Grillo"2 (1949-1966). Fue a través de esta publicación que ellas se encargaron de difundir los hallazgos y objetos arqueológicos recolectados en las salidas de campo organizadas por Francisco Oliveras (Figura 3), así como otros objetos de origen prehispánico originarios de distintas partes del continente americano (Bandrymer y Pontet, 2006).

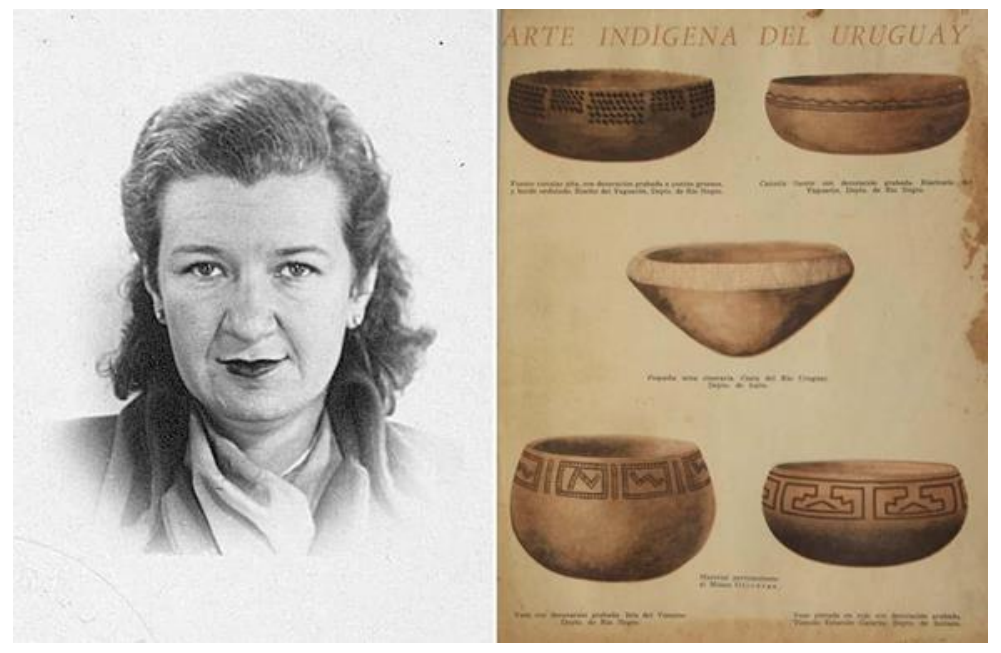

Figura 3. Izq. Bell Clavelli. Año 1951. Fuente: Familysearch.org. Der. Ilustración de materiales arqueológicos cerámicos. Fuente: Revista El Grillo $N^{\circ} 7$ (Consejo Nacional de Enseñanza Primaria y Normal, 1950:15).

La colección, que hasta el día de hoy lleva el nombre de "Francisco Oliveras", fue donada al Estado en 1976, y constituyó la base para la creación en el año 1981 del Museo Nacional de Antropología de Montevideo (Ley Nacional $\mathrm{N}^{\circ}$ 15.167) (MNA, s.f. a). A partir de esta creación, Bell Clavelli fue designada como la primera Directora del Museo Nacional de Antropología, ejerciendo este rol hasta su fallecimiento en 1990 (MNA, s.f. a). No obstante esto, su mención como Directora no ha sido tan ampliamente visibilizada como sí lo fue el papel de su esposo como creador de la colección. Hasta el momento, en la web del MNA, no figura su período como Directora: solo se hace referencia a la donación de la colección por parte de su marido y a que una de las subcolecciones del MNA recibe su nombre. Tampoco hay datos personales o de su trayectoria laboral en las reseñas biográficas del Museo Nacional de Historia Natural (antiguamente asociado al MNA), a diferencia de los de su marido y de otras personalidades 
destacadas por la institución (MNHN, s.f.). Incluso, su nombre no aparece en los créditos de la publicación de la Primeras Jornadas de Ciencias Antropológicas en el Uruguay organizadas por el Ministerio de Educación y Cultura, ni se cuenta con una presentación realizada por ella. Solo se encuentra una mención muy puntual realizada por Arturo Toscano en relación a la gestión del museo (Toscano, 1989: 281). El ínfimo ${ }^{3}$ lugar donde se ofrece información un poco más extensa sobre la vida y obra, así como palabras alusivas a los aportes de Bell, es en un panel ubicado en una de las paredes laterales del zaguán de entrada del museo, donde se la destaca como:

“... compañera incondicional que cumplió un papel fundamental en el desarrollo y conservación de la Colección Oliveras. Su profundo conocimiento de la Colección ameritó que el Ministerio de Educación y Cultura la designara como Directora del nuevo Museo Nacional de Antropología en el momento de su fundación" (Cursivas de las autoras).

Asimismo, se colocó una cita del Prof. Francisco Oliveras en la cual señala lo siguiente (Cursivas de las autoras):

"En mi vida he tenido generosos apoyos, ayudas y estímulos descollando sobre todo ello la vocación, abnegación, dedicación y renunciamiento totales de mi esposa Prof. Bell Clavelli Gamberoni de Oliveras quien hizo posible gran parte de mi obra y que fue y es la eficientísima organizadora de las salas del Museo".

Sin embargo, cabe señalar que la figura de Bell ha sido reconocida $\mathrm{y}$ revalorizada desde el propio Museo Nacional de Antropología a través del diseño de una exposición temporal titulada "Miradas. Pioneras de la arqueología uruguaya" 4 , pensada para el mes de marzo de 2021 pero que tuvo que ser suspendida debido al cierre como medida de control del avance de la pandemia provocada por la COVID-19 ${ }^{5}$. No obstante, más allá de su inauguración, esta exposición constituye, sin dudas, un primer paso en el camino hacia la visibilización de la labor de Bell y de sus aportes a la conservación de las colecciones del museo y a la socialización del conocimiento arqueo-antropológico.

\section{Las mujeres de la Sociedad de Amigos de la Arqueología}

La Sociedad de Amigos de la Arqueología fue constituida el 29 de junio de 1926 con el propósito de "agrupar en torno suyo todos los elementos que en el país se sintieran inclinados al culto de las cosas arcaicas" (Sociedad de Amigos de la Arqueología [SAA], 1927: 5). De acuerdo a Lezama (1995: 1), la creación de esta 
Asociación fue la culminación de un proceso, vinculado al incremento en los intereses culturales dentro de la sociedad uruguaya, la difusión de los conocimientos y la educación primaria obligatoria, que crearon un ambiente propicio en el marco del cual surgieron muchos aficionados a la arqueología, se comenzaron a formar colecciones y a publicar sobre estos temas. En el momento de su creación, la asociación estaba compuesta por 53 socios, todos hombres (SAA, 1927: 226).

En 1927 se publicó el primer número de la Revista de la Sociedad de Amigos de la Arqueología. En los años iniciales fue una sociedad muy activa y editó un volumen de su revista cada año, aunque luego se fueron espaciando y el último tomo (XVII) se publicó en 1978, como cierre de las actividades de la SAA.

\section{Primeras socias de la Sociedad de Amigos de la Arqueología}

En la sesión directiva del 5 de agosto de 1927 se aprueba por primera vez el ingreso como socias de la SAA de dos mujeres: la Srta. Micaela Baraibar y la Sra. Antonia Nuñez de Fernández (SAA, 1927: 252). Ambas socias pioneras eran maestras de profesión, y si bien es aún escasa la información que tenemos sobre ellas, se debe destacar que fueron las dos primeras mujeres que aparecen, oficialmente, en un mundo que era, hasta entonces, exclusivamente de hombres.

Micaela Baraibar, nació en el departamento de Colonia, Uruguay en 1895. Ingresó a la SAA cuando era soltera. Poco después, en 1931, contrajo matrimonio, luego de lo cual tuvo cuatro hijos. Dejó de figurar como socia a partir del año 1932.

Antonia Núñez ingresó casada y aparece como socia hasta 1931. Fue maestra y luego inspectora de educación primaria en el departamento de Salto.

Para 1928 la SAA ya contaba con 90 socios activos de los cuales sólo cuatro eran mujeres. A las ya citadas Micaela Baraibar y Antonia Núñez de Fernández, se suman las "señoritas" Margarita Sabater y Elisa Peña, esta última de Buenos Aires (SAA, 1928).

En el IV tomo de la Revista de la SAA editado en 1930, se menciona por primera vez a una socia correspondiente, la primera mujer extranjera que se vincula a la SAA, Mme. R. Livillier, de Viena. Creemos que hace referencia a Juana de Levillier, esposa de Roberto Levillier, un diplomático argentino que se vincula con la SAA. En la edición de los tomos $\mathrm{V}$ y siguientes, aparece él como socio correspondiente desde Praga, en los tomos VIII-X desde Montevideo, y en el tomo XI desde Buenos Aires. Uno de los varios documentos del archivo consultado que atestiguan los viajes realizados por los Levillier, menciona que realizaron un viaje desde Southampton hacia Nueva York. Juana o Jean Levillier como la denomina, 
era de origen escocés, nacida en Jodhpur, India, en 1893, durante el período de dominio británico en el país.

Hasta el IV tomo, a pesar de que habían comenzado a integrarse varias socias, no había ninguna publicación de autoría femenina. El primer texto escrito por una mujer aparece en este tomo, pero no refiere a ningún tema vinculado con la arqueología sino que se trata de una poesía de Juana de Ibarbourou (SAA, 1930: 345) dedicada al "Higuerón de la Agraciada", árbol de tradición centenaria vinculado al evento histórico del desembarco de los 33 Orientales, que la SAA velaba por conservar y junto al cual se colocaron sendas lápidas, una con la poesía de Juana de Ibarbourou y otra con palabras de Juan Zorrilla de San Martín (SAA, 1930).

En 1931 ingresaron dos nuevas socias, ambas provenientes de Carmelo, Colonia: la Srta. Amelia Forets y la Sra. Lola S. de Indart. Para este año el total de socios asciende a 186 de los cuales apenas seis son mujeres (SAA, 1931, tomo V) y no hay cambios con respecto a las socias en el siguiente año (SAA, 1932, tomo VI). Luego, en el tomo VII de 1933, aparece la participación de una nueva mujer extranjera entre 22 socios correspondientes, la Sra. Matilde Dolgopol de Sáez, de La Plata, Argentina, quien ingresa junto a su esposo Francisco Alberto Sáez.

Durante varios años no se notan cambios en la SAA en cuanto a las mujeres socias, quienes, en muchos casos, son familiares (esposas, hijas o hermanas) de socios varones y se mantienen en franca minoría dentro del total. Cabe señalar además, que la participación en la SAA de esas pocas mujeres está acotada en el tiempo a unos pocos años y no hay trabajos de su autoría.

\section{El comienzo del cambio: Mujeres en la directiva de la Sociedad de Amigos de la}

\section{Arqueología}

En julio 1944 una nueva directiva integra, por primera vez, a una mujer dentro de los 10 titulares: la Sra. María Teresa P. B. de Pereyra es electa como 5a Vocal de la directiva en funciones en el período 1944-1946. Había sido admitida como socia en enero de ese mismo año (SAA 1947: 323). María Teresa P. B. de Pereyra era la viuda del socio Armando Pereyra según se deja constancia en las actas de la asociación, que afirmaban que ella era una continuadora de su labor:

"Voy a agregar aún el nombre del Prof. Armando Pereyra, novel y entusiasta investigador que en una expedición en busca de piezas etnográficas hallara muerte trágica, ahogándose en las aguas del Río Uruguay. Su señora, actual socio $^{6}$ de esta Institución, prosigue la labor iniciada y se halla dotada de un enorme entusiasmo por estas disciplinas" (SAA, 1947: 324). 
La siguiente directiva (1946-1948) vuelve a estar integrada exclusivamente por hombres, situación que se continúa por varias directivas más.

\section{Violeta Bonino de Langguth, 'una mujer con mucha garra'}

Violeta Bonino nació en Montevideo en 1913 y falleció en el año $1987^{7}$. Era arquitecta de profesión, pero realizó investigaciones en diversas ciencias, entre las que destacamos arqueología, paleontología y malacología. Fue una mujer activa en varias instituciones científicas tanto en la investigación como en la gestión, así como docente de la Facultad de Humanidades y Ciencias (MNA, s.f. b).

En 1956 se integra a la Sociedad de Amigos de la Arqueología, junto a su esposo Alfredo Langguth, pero en este caso es ella quien detenta un puesto de suplente en la directiva entre 1956-1958 (SAA, 1956: 504). Su participación es muy comprometida dentro de la asociación y según las actas de este año siempre está presente en las reuniones de directiva.

En marzo de 1957, renuncia a su cargo el secretario Raúl Santiago Acosta y Lara (SAA, 1957: 469) y en su lugar asume la secretaría de la Sociedad de Amigos de la Arqueología Violeta Bonino, actuando en ese cargo por primera vez el día 8 de mayo de 1957. En esa misma reunión, se deja constancia del informe que Violeta presenta sobre las salidas llevadas a cabo "por el Centro de Estudios de Ciencias Naturales al Cabo Polonio, en la costa atlántica, en Rocha, y a la Sierra de la Aurora, en Rivera, exposición que es oída con el mayor interés por los presentes motivando interesantes dialogados." (SAA, 1957: 472).

Podemos destacar dentro de su actuación, que fue la primera mujer que publicó un artículo de su autoría dentro de la Revista de la Sociedad de Amigos de la Arqueología, en su tomo XV editado en 1957, en el que rinde homenaje a Florentino Ameghino a 80 años de que él escribiera el texto Sobre Antigüedades Indias de la Banda Oriental (Bonino de Langguth, 1957).

Desarrolló investigaciones en temas específicos y realizó publicaciones que al día de hoy continúan siendo citas en sus áreas de interés. En 1961 publicó Los primitivos habitantes del Uruguay, y el Uso de los Moluscos en su Economía, en su Decoración y en sus Ritos, en la publicación de la Sociedad de Malacología del Uruguay (Bonino de Langguth, 1961). También realizó investigaciones sobre materiales arqueológicos óseos labrados provenientes de Colonia Concordia, en Soriano y presentaciones en los congresos de la época, como el V Encuentro de Arqueología del Litoral, en Fray Bentos, Uruguay en 1977, en cuyas Actas publicó uno de sus artículos (Bonino de Langguth, 1977). Investigó sobre la pesca indígena en la zona de Colonia, tema sobre el que escribió el artículo Pesas para redes de 
presunta procedencia indígena usadas por los primitivos habitantes de la ciudad de Colonia del Sacramento (Bonino de Langguth, 1982).

Violeta Bonino falleció el 25 de abril de 1987 y es recordada con cariño por colegas de la Sociedad Uruguaya de Malacología en un obituario que denota el respeto que inspiraba y lo activa que era su participación tanto en las reuniones como en salidas de campo, en las que recorrió casi todo el país junto al grupo de investigación de Ciencias Naturales. Asimismo, es recordada su labor docente en la cátedra de paleontología de la Facultad de Humanidades y Ciencias (Sicardi, 1989 [1987]: 2).

Años más tarde, en 2019, su hija donó al Museo Nacional de Antropología su colección arqueológica (MNA, 2019).

Para culminar este apartado sobre la figura de Violeta Bonino, debemos resaltar que en las entrevistas realizadas a las primeras egresadas de la carrera, el suyo fue un nombre que surgió como una referente entre las mujeres, como alguien que las estudiantes de la primera generación veían con admiración y respeto por su labor profesional:

“...yo quiero llegar a defender así mis ideas, es Violeta Bonino de Langguth. (...) tú la veías en los congresos y era una mujer grande, [...] pero era una mujer con mucha garra, ...plantada en un mundo de hombres, porque había muy pocas mujeres, la única quizás en esos momentos. [...] como mujer, yo con la que me quedé con esa garra que tenía para presentar y era sola ella, la única mujer era ella, Violeta. De esa gente que decís "yo quiero ser como ella"." (Entrevista a B., 24/02/21).

\section{Las mujeres integrantes del Centro de Estudios Arqueológicos}

\section{(CEA)}

El Centro de Estudios Arqueológicos (CEA) fue una institución privada que desarrolló sus actividades desde 1969 y tuvo personería jurídica hasta 1992 (Jorge Baeza y Wilder Melgar, com. pers. febrero 2021). Su última Comisión Directiva (período 1991-1993) estaba integrada por tres hombres (Jorge Baeza en la Dirección, Milton Pinto y Wilder Melgar) y cuatro mujeres (Alba González como Secretaria General, Celia Arbon, Blanca R. Domínguez y Silvia Varela), además de cinco mujeres y dos hombres como suplentes (Centro de Estudios Arqueológicos [CEA], 1991). Convivió con la creación de la carrera de arqueología, y según Cabrera Pérez (2011: 93) marcó la etapa de consolidación de la disciplina en nuestro país. 
Sus integrantes se reunían una vez por semana en el Museo Nacional de Historia Natural (MNHN) y sus fines se orientaban al estudio científico de la Arqueología Americana y de la Prehistoria del Uruguay, la promoción de la enseñanza de la Arqueología nacional, así como su difusión a través de publicaciones, exposiciones y conferencias (CEA, 1989). El CEA también buscó el intercambio y la cooperación con distintas instituciones culturales y académicas uruguayas y extranjeras, y fue muy importante el intercambio con los y las profesoras visitantes (CEA, 1989).

En el CEA participaron alrededor de quince mujeres ${ }^{8}$ que han contribuido al desarrollo de la arqueología en una variedad de actividades tales como trabajos de campo y laboratorio, publicaciones y organización y participación en congresos. Además, dentro de los seminarios de formación y actualización organizados por el CEA entre 1972 y 1987, se contó con la participación de arqueólogas extranjeras tales como la Dra. Antonia Rizzo (Universidad Nacional de La Plata, Argentina), la Dra. Betty Meggers (Instituto Smithsoniano de Washington, EE.UU.), la Dra. Ítala Basile Becker (Instituto Anchietano de Pesquisas, Universidade de Vale do Rio dos Sinos, Brasil), la Lic. Silvia Álvarez (Escuela Superior Politécnica del Litoral de Guayaquil, Ecuador) y la Lic. Marta Páez (Universidad Nacional de Mar del Plata, Argentina) (CEA, 1989).

Unas de las primeras consideraciones y discusiones que tuvimos fue el mantener el "de" en las fichas biográficas y en las presentaciones. Algunas de ellas, a lo largo de su carrera fueron abandonando el "de", otras sin embargo, nos indican: 'te pido que pongan el 'de Pinto', porque mi marido era más conocido que yo" (Silvia Varela, com. pers. marzo 2021). Esta asunción, o mismo precepto, de que el marido o el compañero fue el experto aparece recurrentemente en las entrevistas:

"Llegué [a la arqueología] por casualidad porque mi marido había ido de pesca al Paso de los Negros, y ahí en una barranca encontraron una urna guaraní [...] y a partir de ahí nos hicieron la conexión con el Museo [Histórico de Durazno] [...] y de ahí surgió todo el interés, y realmente el arqueólogo fue mi marido, yo colaboraba, ayudando..." (Entrevista a A., 5/11/2020).

Es además una idea que se extiende más allá del CEA:

"Y las mujeres que había por ejemplo en el Centro de Estudios Arqueológicos, en el CEA, había algunas que estaban como Mabel Moreno, que después llegó mucho más [...], pero después otras mujeres eran más 'esposas de', que (...) no eran referentes" (Entrevista a B., 24/02/21). 
Estas mujeres que hoy tienen alrededor de 70 años eran muy jóvenes cuando se empezaron a vincular al CEA. Si bien realizaron salidas de campo, pocas recuerdan hoy lugares específicos o sitios. Participaron activamente de las charlas organizadas por el CEA, aunque sus actividades principales consistieron en la organización de los congresos, "papelería" y dictado de cursos.

De las entrevistas realizadas se extrae que estas mujeres no desarrollaron lazos de amistad o sororidad entre sí. Tampoco recuerdan especialmente a las arqueólogas provenientes de otros países. En la consulta sobre referentes mencionan principalmente varones, en especial a Antonio Taddei fundador y primer presidente del CEA, y en un caso destacan a la arqueóloga argentina Antonia Rizzo ${ }^{9}$. Las arqueólogas visitantes y participantes de los congresos regionales las recuperamos de los documentos y publicaciones; entre estas se encuentran María Amanda Caggiano (1977), Betty Meggers y Jeanne Stanford (1977) e Ítala Basile Becker (1972, 1973, 1976) (Museo Municipal de Historia Natural de Río Negro [MMHNRN], 1973, 1975; MEC, 1977). En relación con esta última, la visita a nuestro país de Ignacio Schmitz e Ítala Basile Beker será un antecedente importante del desarrollo de la arqueología en nuestro ámbito (Cabrera Pérez, 2011: 93) e incluso se fijan fechas de congresos en relación a su venida (MMHNRN, 1973:1).

Para estas jóvenes también tendría influencia la figura de Annette LamingEmperaire, francesa, nacida en Petrogrado el 22 de octubre de 1917 y que falleció en Curitiba, en mayo de 1977 tras un accidente. Annette ${ }^{10}$ estuvo en Uruguay en ocasión de la organización de la Misión de Rescate Arqueológico de Salto Grande (MRASG), financiada por UNESCO y de la que fue Directora desde 1976 a 1977 (fecha de su muerte).

“...[Referente] Por su historia de vida, durante la guerra, por saber, por poder conjugar un marido arqueólogo con ella arqueóloga, por seguir adelante después de muerto el marido, por sus trabajos, su librito [...] qué claro que explicaba para esa época donde la arqueología prehistórica había que hacerla en un libro de difusión, que lo usábamos nosotros también, ¿no? porque era la... y después Mary Leakey eran las mujeres que veías, pah, mirá lo que hicieron, ¿no? pero, acá no había referentes mujeres, no había, porque obviamente, estábamos todos empezando" (Entrevista a E., 24 de febrero de 2021).

Tras el fallecimiento de Annette Laming-Emperaire, se encarga a la Dra. Niède Guidón nacida en Jaú, estado de São Paulo, el 12 de marzo de 1933, la Dirección de la Misión de Salto Grande. Niède será Directora desde 1977 a 1983 (Guidón, 1977, 1987). Ya en 1973 la brasilera forma parte de la Misión Arqueológica Franco-Brasileña que inicia las investigaciones arqueológicas en 
Piauí. Será la responsable de la edición de los informes y volúmenes correspondientes a los resultados del rescate arqueológico. Sin embargo, aparentemente, el contacto con las estudiantes uruguayas habría sido muy limitado ya que los equipos nacionales de la Universidad dirigidos por el Dr. Austral y el equipo internacional tuvieron poco contacto entre sí y tanto las excavaciones como las instancias de laboratorio se hicieron de forma separada.

Un hito en la participación de las mujeres en la arqueología será el desarrollo de los primeros congresos nacionales ${ }^{11}$, en los que estas "mujeres de" empiezan a aparecer en las publicaciones como autoras. Son los primeros trabajos de Mabel Moreno y Silvia Varela (Bosch et al. 1973), Cristina Rouco (Díaz Rodríguez y Rouco, 1973; Rouco et al., 1973). En ese mismo primer congreso nacional presentará también Leila Tuya el Informe sobre el material cerámico de la Colección Maeso (Tuya de Maeso, 1973).

Una mención especial merece el desarrollo del $\mathrm{V}$ Encuentro de Arqueología del Litoral del 30 de octubre al 2 de noviembre de 1977 en Fray Bentos, con amplia presencia femenina no sólo como expositoras sino también como asistentes, en especial las nóveles estudiantes de la carrera (MEC, 1977) (Figura 4). En esta reunión se presentaron 21 trabajos, seis de los cuales fueron presentados en coautoría de mujeres y hombres, siete en autoría o co-autoría de varones y ocho trabajos en autoría y coautoría de mujeres. Tres de estos últimos corresponden a trabajos de la entonces Licenciada María Amanda Caggiano del Museo de La Plata (Caggiano, 1977 a y b; Caggiano y Fernández, 1977) y dos de la Dra. Antonia Rizzo de la Facultad de Ciencias Naturales y Museo, La Plata (Cione et al., 1977; Rizzo, 1977).

Uruguay participó con los trabajos arqueológicos del CEA sobre cerámica del Río Tacuarembó y Río Negro con autoría, entre otros, de Mabel Moreno (Bosch et al., 1977); sobre Salto Grande -con dataciones por radiocarbono- bajo la autoría, entre otros, de Marita Fornaro (Baeza et al., 1977); y sobre investigación del arte rupestre con autoría de Mario Consens y Yubarant Bespali de Consens (1977). Fornaro y Antonio Díaz, por la Facultad de Humanidades y Ciencias, expusieron un trabajo sobre cerámica del litoral (Díaz y Fornaro, 1977). Fue presentado también el trabajo ya mencionado de Violeta Bonino (1977) sobre enderezadores de flechas del litoral del río Uruguay. Ítala Basile Becker presentó, junto con Juana Paris de Cebey (1977) de la Facultad de Humanidades, el trabajo Os indios da Banda Oriental.

Algunas de las mujeres que integraron el CEA continuaron su formación universitaria cuando se creó, en 1976, la Licenciatura en Ciencias Antropológicas en la Universidad de la República. Tanto Marita Fornaro como Mabel Moreno se recibieron en Antropología Social. Sara Campos prosiguió sus estudios en 
Arqueología y, aunque no llegó a completar su título, es pionera en Uruguay de los estudios de paleobotánica. Otras siguieron su interés en la arqueología desde otros lugares y ámbitos, como por ejemplo el Grupo de Estudios de Reconocimiento Geográfico del Uruguay (GERGU).

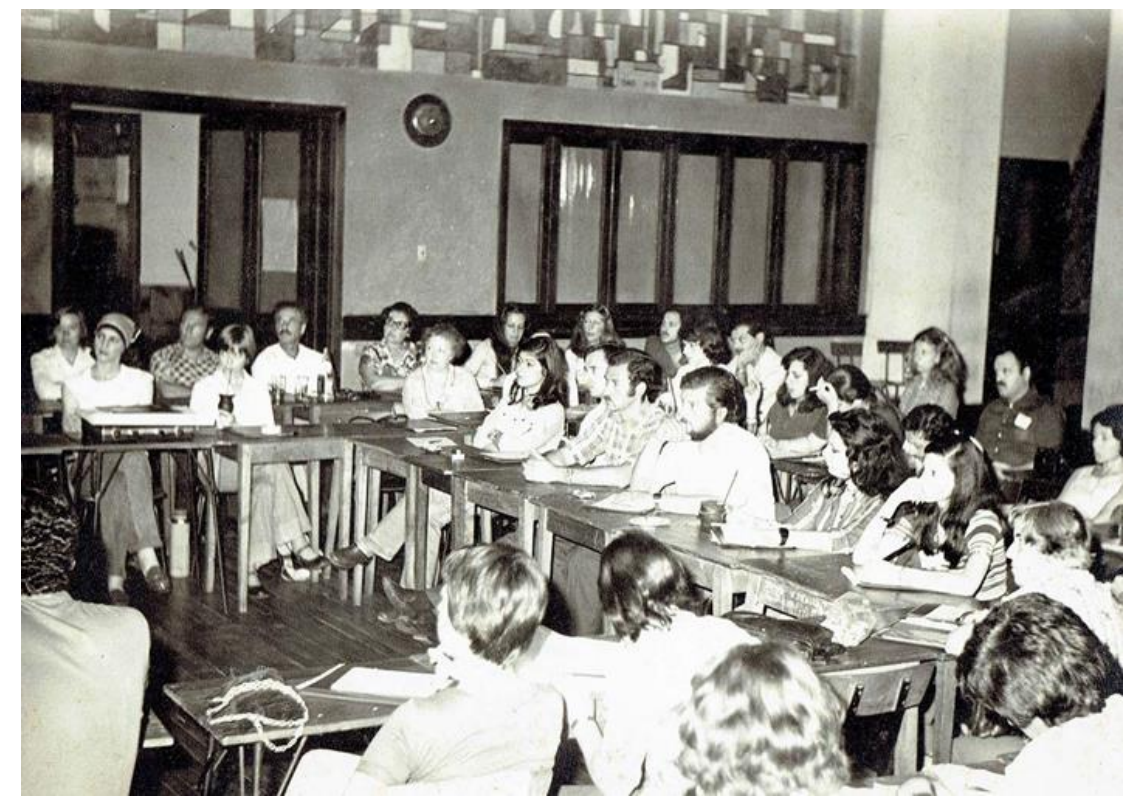

Figura 4. V Encuentro de Arqueología del Litoral, octubre-noviembre de 1977, Fray Bentos. Fotografía cedida gentilmente por Mónica Sans.

\section{Las primeras egresadas}

En 1976 se crea en la Facultad de Humanidades y Ciencias la Licenciatura en Ciencias Antropológicas, con una especialización en Arqueología y Prehistoria. Varias mujeres pertenecieron a esta primera generación de arqueólogas y han desarrollado una importante trayectoria de docencia, gestión e investigación con distintas especialidades. Algunas de ellas son: Mónica Sans, Nelsys Fusco, Elianne Martínez, Carmen Curbelo y Susana Cavellini.

Estas mujeres participaron, desde los inicios de sus carreras, en las primeras excavaciones lideradas por arqueólogos y arqueólogas extranjeras, principalmente en la MRASG (Comisión UNESCO) dirigida por Annette LamingEmperaire y Niède Guidón, así como en los trabajos de laboratorio que acompañaron y siguieron a las actividades de campo. También asistieron a los encuentros organizados por el CEA (en la Figura 4 se puede observar, por ejemplo, a Mónica Sans). 
En 1986 el Ministerio de Educación y Cultura (MEC) crea la Comisión de Rescate Arqueológico de la Cuenca de la Laguna Merín (CRALM), la que marcó el inicio de una intensa actividad de investigación sobre los cerritos de indios de las tierras bajas del este de Uruguay con el fin de paliar la situación de la pérdida de sitios arqueológicos a raíz de las canalizaciones por la fuerte expansión del cultivo de arroz (Durán Coirolo y Bracco Boksar, 1996). Las actividades arqueológicas desarrolladas en este marco contribuyeron con la formación académica de las primeras generaciones de estudiantes y egresadas de la Licenciatura.

Todas ellas también participaron de la Asociación Uruguaya de Arqueología (AUA) fundada en 1995. Fueron socias fundadoras de la asociación y participaron en la Comisión Directiva y Comisión Fiscal en diversos períodos.

Algunas de las primeras egresadas de la carrera ingresaron por concurso al plantel docente de la Facultad de Humanidades y Ciencias de la Educación. Otras se integraron a instituciones públicas tales como diversas dependencias del MEC, entre ellas, la Comisión de Patrimonio Cultural de la Nación (CPCN) y los museos de Antropología y de Artes Decorativas.

Es de destacar, entre otras cuestiones, que ellas impulsan en esos primeros años trabajos de socialización del conocimiento arqueológico (p.e. Cavellini, 1992, 1993, 1994; Curbelo et al., 1987; Fusco, 1989; Martínez et al., 1989). Como plantea una de ellas:

“... nos íbamos dando cuenta, porque recién seríamos posiblemente diez arqueólogos recibidos, la necesidad de poder difundir la importancia de proteger los sitios y ahí hacemos un proyecto con primaria muy interesante, que todos lo recuerdan hasta ahora, que fue llegar a todas las escuelas rurales y suburbanas del país con un proyecto para proteger los sitios arqueológicos prehistóricos" (Entrevista a C., 5 de noviembre de 2020).

Mónica Sans y Nelsys Fusco fueron en 1983 las dos primeras egresadas de la Licenciatura en la rama de Prehistoria y Arqueología. Sus trayectorias personales fueron diferentes, una se orientó como estudiante a la Antropología Biológica y la otra tuvo una clara preocupación por los temas de gestión.

Mónica Sans comenzó a interesarse por el material óseo desde su primera excavación en Salto Grande. Poco después de finalizada la carrera, ingresó a trabajar con un cargo docente en la Facultad de Humanidades y Ciencias y decidió especializarse en el tema de la Antropología Biológica que hasta ese momento no tenía ningún desarrollo en el país. Se formó con diversos investigadores extranjeros, decidió quedarse en Uruguay y realizar su maestría y doctorado en Ciencias Biológicas por el Programa de Desarrollo de las Ciencias Básicas 
(PEDECIBA), de la Universidad de la República. Fue la impulsora en el marco de la Licenciatura en Ciencias Antropológicas, primero de un área, y luego de un departamento de Antropología Biológica dentro del instituto de Antropología, donde se realizan investigaciones en poblaciones antiguas y modernas, abordando diversos tópicos como genética de poblaciones, bioarqueología, biodemografía, etc. Dentro de sus mayores logros académicos, ella destaca que a través de las investigaciones llevadas a cabo por su equipo en Uruguay se pudo dar a conocer lo que hasta entonces se negaba, dando visibilidad al tema indígena y su continuidad en la población actual.

Nelsys Fusco primero se formó como maestra especializada en Ciencias Sociales y luego como arqueóloga. Comenzó participando de excavaciones de sitios prehistóricos y luego se especializó en Arqueología Histórica. Realizó estudios de posgrado, por un lado vinculados a la cerámica y a la Arqueología Histórica en Estados Unidos (University of Florida) y, por otro, en patrimonio cultural y turismo, en la cátedra UNESCO de Turismo Cultural de Buenos Aires. Desarrolló sus intereses tanto en la investigación como en la difusión del patrimonio. Se radicó en Colonia de Sacramento e investigó sobre la cerámica Faiança portuguesa. Desde 1983 fue arqueóloga asesora de la oficina de la Comisión de Patrimonio Cultural de la Nación (MEC) - Sede Colonia hasta 2020.

$\mathrm{Al}$ año siguiente, en 1984, se graduaron también Susana Cavellini, Carmen Curbelo y Elianne Martínez. Las dos últimas junto a Nelsys Fusco y Leonel Cabrera Pérez ingresaron a la Comisión de Patrimonio, y realizaron entre otros las investigaciones arqueológicas en Isla Gorriti. Posteriormente, el MEC creó la Comisión de Arqueología, ámbito al que se trasladaron Cabera y Curbelo, y Fusco y Martínez continuaron en la primera.

En 1977, cuando era estudiante de Antropología, Susana Cavellini formó parte del equipo que trabajó en la MRASG y en 1978, con 22 años, viajó a España, Portugal e Italia con el fin de investigar las fuentes existentes en los archivos históricos para UNESCO, trabajo que la convirtió en una de las pioneras en el desarrollo de la etnohistoria en el Uruguay. Más adelante participó en numerosas investigaciones arqueológicas de la ciudad de Colonia del Sacramento. Desde los inicios de su carrera profesional trabajó en el campo museológico. Fue de las primeras en aplicar la moderna teoría y metodología en museología y museografía en nuestro país para colecciones arqueológicas, que se vio reflejada en trabajos tales como el diseño de la primera exposición de materiales arqueológicos procedentes de excavaciones locales en Colonia y en la exposición de la Colección de Arqueología Clásica y Musulmana en el Museo de Artes Decorativas de Montevideo. Cuando era Directora de este último generó, además, una sala de laboratorio y reserva dentro del museo, visible para el público (única en el país). 
Carmen Curbelo, en los primeros años de su carrera profesional y académica (1986 y 1991) fue corresponsable científica dentro de la CRALM de las investigaciones en Laguna Merín. Posteriormente, entre 1991 y 2000 también dentro de la Comisión de Arqueología del MEC, inició su trayectoria en Arqueología Histórica como corresponsable de los trabajos en la Bahía de Maldonado, en el marco de los cuales realizó investigaciones arqueológicas en Isla Gorriti (Batería de Santa Ana) y en la costa de la Bahía (Batería de Jesús), que luego derivaron en otros proyectos en la ciudad. Ha investigado sobre temas tales como arqueología del contacto afro-americano, arqueología industrial y, principalmente, arqueología indígena-misionera. Esta línea que comenzó en 1995 continúa hasta el día de hoy con su proyecto de Dedicación Total (FHCE, Udelar). Ha trabajado en distintas localidades del país y ha realizado estudios de impacto arqueológico y distintas consultorías públicas y privadas. También realizó trabajos en la Provincia de Corrientes (Argentina).

Elianne Martínez también participó como estudiante de la carrera en Antropología en la MRASG colaborando en el Laboratorio de Salto Grande en Montevideo principalmente en análisis lítico. Participó también de las excavaciones en Paypaso dirigidas por Antonio Austral. En 1985, recientemente recibida, fue co-responsable de las investigaciones en Isla Gorriti. Desde el Departamento de Arqueología de la CPCN Elianne se volcó tempranamente a la labor como gestora patrimonial. Su carrera será marcada por la amenaza de la construcción de un Hotel 5 estrellas en la Isla Gorriti y el interés en investigar el área. Finalmente, la construcción se logró detener. Un poco más tarde también se vinculará con el tema de la arqueología subacuática y la del patrimonio en el área protegida de Chamangá, Departamento de Flores. Actualmente es la encargada del Departamento Técnico de Arqueología de la CPCN.

\section{REFLEXIONES FINALES}

Nuestra intención es dar visibilidad a las mujeres en la historia de la arqueología del Uruguay y, sin proponérnoslo, a través de esto comenzamos a andar un camino que no tiene vuelta atrás. Esto es nuevo para nosotras y creemos que forma parte de un movimiento mayor en el que están involucradas otras colegas y distintas líneas de investigación. La arqueología uruguaya está en un proceso, que entendemos es necesario, de cuestionar los mandatos, las visiones naturalizadas y las desigualdades. En este marco, este artículo es tanto una consecuencia de ese movimiento como un aporte más a dicha construcción. Nuestra propia investigación nos ha sorprendido y ha tenido repercusiones inesperadas entre estudiantes y colegas. Ha permitido compartir entre nosotras, 
reflexionar y cuestionarnos, sobre nuestras propias experiencias, sobre experiencias de otras mujeres vinculadas a la violencia machista, pero también a las formas de relacionarnos entre nosotras las propias arqueólogas.

Nos pusimos los lentes violetas y comenzamos a revisar nuestros propios preconceptos, los cursos y las bibliografías con las que nos formamos. Empezamos a ver entonces cómo estos reflejan una visión androcéntrica por un lado, y por otro, la naturalización de la jerarquización tanto de las figuras como de la producción académica de unos y otras. Surge asimismo, que estas son naturalizaciones de las jerarquías machistas que refieren no solo a la relación entre hombres y mujeres, sino también de todo lo que es visto como académico y dentro de la ciencia, y lo no académico, que se minimiza, se rechaza. Al mismo tiempo, se refleja en una naturalización en las visiones del pasado construidas desde la arqueología en las que se invisibiliza a las mujeres.

Estas últimas líneas son de interés para poder seguir explorando a futuro. A partir de este trabajo, vemos cómo se van abriendo otras líneas conjuntas con otras colegas que transitan caminos paralelos. Y será necesario amplificar las sinergias para seguir avanzando.

En este caso, la publicación en el número monográfico "Género en la Arqueología. Experiencias, análisis y perspectivas futuras”, es una oportunidad para dar a conocer estas historias invisibilizadas, pero también, una invitación a seguir avanzando en la búsqueda de más pioneras.

Lamentablemente, la pandemia de COVID-19 ha restringido el acceso a archivos de museos y personales que estaba planificado relevar. Es el caso por ejemplo del acceso a las Actas y otros documentos del CEA, que se encuentran en el Museo Arqueológico Prof. Antonio Taddei de la Ciudad de Canelones, y documentos del acervo de la Colección Maeso Tognochi del Museo Nacional de Antropología. Otro tanto de documentos y fotografías en archivos personales que vieron retrasado el relevamiento por cuestiones de distanciamiento o cuarentena, o simplemente por temas de cuidados propios de nosotras las autoras. Pese a las dificultades, el realizar este proyecto ha significado una actividad sumamente gratificante, en medio de los impactos que tiene para nosotras como académicas, docentes, madres.

El diálogo intergeneracional que significa la investigación y que esperamos poder continuar, crea además activaciones para una arqueología en el presente. Para nuestras colegas de mayor experiencia, ha significado no sólo recordar sino también cuestionar aquello por lo que habían pasado, repensar las dinámicas, los roles, incluso reconocer aquello que está tan naturalizado. Para las estudiantes, próximas arqueólogas, como comenta una de ellas en clase es "sentir el apoyo y que podamos enorgullecernos entre todas". Para la construcción de una arqueología 
más equitativa, como dice Rebeca Solnit “... por mí, pero también por esas mujeres más jóvenes que tienen algo que decir, con la esperanza de que puedan decirlo." (Solnit, 2019:17).

Para esto también será necesario recuperar los silencios referidos a quienes no pudieron jugar el juego, el registro de a quienes, en palabras de GonzálezRamírez (2020), no les alcanzó con hacer trabajo duro y serio. Esta mirada (desde los cuerpos) requiere analizar la estructura de clase, las jerarquizaciones y la precarización de nuestra disciplina, tanto más en los márgenes territoriales. Estas mujeres también van apareciendo de a poco en las entrevistas:

"Mónica (...) desapareció, no la vimos nunca más" (Entrevista a G., 3 de marzo de 2021).

"Lamentablemente después Juana tuvo que dejar. Tenía toda la carrera hecha" (Entrevista a G., 3 de marzo de 2021).

\section{AGRADECIMIENTOS}

Nuestro más profundo agradecimiento a las mujeres y colegas que accedieron a ser entrevistadas y ser parte de nuestra investigación (Carmen Curbelo, Elianne Martínez, Mabel Moreno, Marita Fornaro, Mónica Sans, Nelsys Fusco, Sara Campos, Silvia Varela). A Ismael Ubilla Cavellini, por las fotografías que nos facilitó de Susana Cavellini. A Carina Erchini, Directora del Museo Nacional de Antropología por la invitación a colaborar con la muestra "Miradas. Pioneras de la Arqueología Uruguaya". A Jorge Baeza y Osvaldo Rodríguez por la información y documentos del CEA. A Aparicio Arcaus por la información y documentos que nos brindó. A Fabrizio Scarabino y Gustavo Tabares por facilitarnos los artículos de la Sociedad de Malacología del Uruguay. A Rafael Suárez por la invitación a participar del curso de Prehistoria del Uruguay, a la que fue nuestra primera clase del proyecto sobre las mujeres en la arqueología, y a las y los estudiantes del curso por el enriquecedor intercambio. Finalmente, a nuestros compañeros y compañeras del LAPPU por el apoyo y permanente discusión que nos alienta a reflexionar sobre nuestra práctica.

\section{REFERENCIAS}

Adovasio, J. M., O. Soffer y J. Page.

2007. The invisible sex. Uncovering the true roles of women in Prehistory. Smithsonian Books/Harper Collins Publishers. Nueva York.
Alberti, B. y V. Williams (eds.).

2005. Género y etnicidad en la Arqueología de Sudamérica. Serie Teórica $\mathrm{N}^{\circ} 4$. FACSO, UNCPBA. Olavarría. 
Arruabarrena, Y. y E. Vallvé.

2009. Carlos Maeso y Leila Tuya. Pioneros de la arqueología uruguaya. Paneles de la exposición permanente del Museo Maeso de Villa Soriano. CPCN. Soriano.

Baeza, J., A. Taddei, J. Femenias, O. Rodríguez, W. Melgar, A. Díaz, M. Fornaro.

1977. Investigaciones arqueológicas en el área de Salto Grande: tres primeros radiocarbonos. En $V$ Encuentro de Arqueología del Litoral: 67-88. MEC. Montevideo.

Bandrymer, S. y R. Pontet.

2006. Aportes desde la microhistoria: el grupo del Centro de Estudios de Ciencias Naturales y las Maestras Pintoras. Imaginarios Prehispánicos en el Arte Uruguayo: 18701970: 113-117. Museo de Arte Precolombino e Indígena. Montevideo.

Basile Becker, I. y J. Paris de Cebey.

1977. Os indios da Banda Oriental - Charrúa e minuano: historico, abastecimento e assentamento sua relação com as frentes de expansão. En $V$ Encuentro de Arqueología del Litoral: 89-110. MEC. Montevideo.

Bonino de Langguth, V.

1957. Sobre Antigüedades Indias de la Banda Oriental. Revista de la Sociedad de Amigos de la Arqueología Tomo XV: 433- 444.

Bonino de Langguth, V.

1961. Los primitivos habitantes del Uruguay, y el Uso de los Moluscos en su Economía, en su Decoración y en sus Ritos. Comunicaciones de la Sociedad de Malacología del Uruguay 1 (1): 11-14.

Bonino de Langguth, V.

1977. Enderezadores de flechas del litoral del río Uruguay. En $V$ Encuentro de Arqueología del Litoral: 113-116. MEC. Montevideo.

Bonino de Langguth, V.

1982. Pesas para redes de presunta procedencia indígena usadas por los primitivos habitantes de la ciudad de Colonia del Sacramento (Uruguay). En Actas del VII Congreso Nacional de Arqueología: 8-10. Impresora Uruguaya SRL. Montevideo.

Bosch, A., M. Moreno de Bosch, M. Pinto y S. V. de Pinto.

1973. Informe sobre la zona Costera Atlántica de Cabo Polonio y Balizas. Intento de reconstrucción arqueológica. 1ra. Parte. En Anales del $2^{o}$ Congreso Nacional de
Arqueología: 171-214. Río Negro, Fray Bentos, Uruguay.

Bosch, A., M. Moreno de Bosch, J. Campos y J. Femenías.

1977. Técnicas y motivos decorativos de la cerámica arqueológica de los Ríos Tacuarembó Grande, Chico y Río Negro Medio (R.O. del U.). $V$ Encuentro de Arqueología del Litoral: 245-261. MEC. Montevideo.

Brum, L.

2008. Prácticas funerarias y complejidad emergente en el este uruguayo (departamento de Rocha). Tesis de Licenciatura, Facultad de Humanidades y Ciencias de la Educación, Universidad de la República. Montevideo, Uruguay. Inédita.

Colegio de Arqueólogas y Arqueólogos de Chile.

S.f. Arqueología y feminismo: la organización como base para los futuros cambios en materia de género. Recuperado en 22 de abril de 2021 de: https://colegiodearqueologos.cl/arqueologiafeminismo-la-organizacion-base-los-futuroscambios-materia-genero/

Cabrera Pérez, L.

1988. Panorama retrospectivo y situación actual de la Arqueología uruguaya. Universidad de la República. Montevideo. Ms.

Cabrera Pérez, L.

2004. Marcos teóricos y criterios dominantes en las tipologías líticas uruguayas. En Politis, G. y R. Peretti (eds.) Teoría arqueológica en América del Sur: 185-196. INCUAPA. Olavarría.

Cabrera Pérez, L.

2011. Patrimonio y Arqueología en la región platense. Departamento de Publicaciones, Unidad de Comunicación de la Universidad de la República. Montevideo.

Cabrera Pérez, L. y C. Curbelo.

1992. Patrimonio y arqueología en el Uruguay: Hacia el reconocimiento de un pasado olvidado. En Politis, G. (ed.) Arqueología en América Latina Hoy: 45-56. Biblioteca Banco Popular, Col. Textos Universitarios. Bogotá.

Caggiano, M. A.

1977 a. Breve reseña de una pesquisa efectuada en el Paraná Ibicuy, Argentina. En V Encuentro de Arqueología del Litoral: 23-25. MEC. Montevideo. 
Caggiano, M. A.

1977 b. Análisis de rasgos decorativos en algunos sitios pertenecientes a la Provincia de Buenos Aires, República Argentina. En $V$ Encuentro de Arqueología del Litoral: 3151. MEC. Montevideo.

Caggiano, M.A. y A. M. Fernández.

1977. Nuevo aporte sobre posibles contactos culturales entre la región andina y el Litoral. En $V$ Encuentro de Arqueología del Litoral: 117-120. MEC. Montevideo.

Cavellini, $\mathrm{S}$.

1992. Arqueología clásica: una experiencia educativa. Primeras Jornadas Nacionales de Antropología. Museo Nacional de Antropología. Separata. MEC. Montevideo.

Cavellini, S.

1993. La socialización del conocimiento de la "prehistoria". En Aportes para el conocimiento de la prehistoria uruguaya: 149-153. MEC. Montevideo.

Cavellini, S.

1994. Arqueología y Educación. Experiencias y reflexiones. En Consens, M., J. M. López Mazz y C. Curbelo (eds) Arqueología en el Uruguay 120 años después. Trabajos presentados en el VIII Congreso Nacional de Arqueología Uruguaya: 371-373. Surcos. Montevideo. Disponible en: https://indiauy.tripod.com/ceci/ceci_a6.htm

CEA.

1989. Centro de Estudios Arqueológicos. Aportes para una Arqueología Nacional (1969-1987). $1^{\text {as }}$ Jornadas de Ciencias Antropológicas en el Uruguay. 23 al 27 de noviembre de 1987: 291-300. Museo Nacional de Antropología. Montevideo.

CEA.

1991. Centro de Estudios Arqueológicos. Boletín Informativo V (1) nov-dic 1991: 1.

Cione, A. L., A. Rizzo y E. P. Tonni.

1977. Relación Cultura indígena-medio ambiente en un sitio de Rincón de Landa, Gualeguaychú, Entre Ríos, República Argentina. Nota preliminar. En $V$ Encuentro de Arqueología del Litoral: 121-141. MEC. Montevideo.

Colomer, L., P. González Marcén, S. Montón y M. Picazo (comp.).

1999. Arqueología y teoría feminista. Estudios sobre mujeres y cultura material en arqueología. Icaria Editorial. Barcelona.
Conkey, M. W. y J. D. Spector.

1984. Archaeology and the study of gender. Advances in Archaeological Method and Theory 7: 1-38.

Consejo Nacional de Enseñanza Primaria y Normal.

1950. El grillo: revista escolar del Consejo Nacional de Enseñanza Primaria y Normal 7. Montevideo.

Consens, M. y Y. Bespali de Consens.

1977. Fundamentos para la aplicación de técnicas documentales en la investigación del arte rupestre. En V Encuentro de Arqueología del Litoral: 143-152. MEC. Montevideo.

Curbelo, C.

2004. Reflexiones sobre el desarrollo del pensamiento teórico en la arqueología uruguaya. En Politis, G. y R. Peretti (eds.) Teoría arqueológica en América del Sur: 259-279. INCUAPA. Olavarría.

Curbelo, C., L. Cabrera, N. Fusco y E. Martínez.

1987. Proyecto de difusión de la Arqueología en el Uruguay. Anales de las Primeras Jornadas de Ciencias Antropológicas en el Uruguay: 307-310. Facultad de Humanidades y Ciencias, Universidad de la República. Montevideo.

Díaz Rodríguez, A. y C. Rouco.

1973. La cerámica de Salto Grande. En Segundo Congreso Nacional de Arqueología del Uruguay: 253-278. CEA. Fray Bentos.

Díaz, A. y M. Fornaro.

1977. Intento de sistematización de las modalidades alfareras del Litoral Uruguayo. En $V$ Encuentro de Arqueología del Litoral: 165174. MEC. Montevideo.

Durán Coirolo, A. y R. Bracco Boksar (eds.).

1996. Arqueología de las Tierras Bajas. Ministerio de Educación y Cultura. Montevideo.

Fusco, N.

1989. La protección del patrimonio prehistórico: Arqueólogos y comunidad desarrollando una propuesta integral. Precirculados de las Jornadas Taller: El uso del pasado: 25-32. Universidad Nacional de La Plata.

Gero, J. M.

1999. Sociopolítica e ideología de la mujer-en-casa. En Colomer, L., P. González Marcén, S. Montón y M. Picazo (comp.) Arqueología y teoría feminista. Estudios sobre mujeres $y$ 
cultura material en arqueología: 341-355. Icaria Editorial. Barcelona.

González-Ramírez, A.

2020. Otras compañeras que no continuaron...Más que olvido, el ojo caníbal. Boletín de la Sociedad Chilena de Arqueología 50: 3-9.

Guidón, N.

1977. Resultados preliminares de la Misión de Rescate Arqueológico de Salto Grande. En $V$ Encuentro de Arqueología del Litoral: 189200. MEC. Montevideo.

Guidón, N.

1987. Salto Grande. Tomos 1 y 2: República Oriental del Uruguay: Misión de rescate arqueológico. MEC. Montevideo.

Lavallée, D.

1978. Annette Laming-Emperaire. Journal de la Société des Américanistes 65: 224-225.

Lezama Astigarraga, A.

1995. Arqueología en el Uruguay. Conferencia dada en la Sociedad de Arqueología Brasilera, Porto Alegre, 11 al 15 de setiembre de 1995. Ms.

López Mazz, J. M.

2004. Arqueología e identidad uruguaya: el saber y el poder en las vanguardias intelectuales. En Politis, G. y R. Peretti (eds.) Teoría arqueológica en América del Sur: 197-211. INCUAPA. Olavarría.

Maeso Tognochi, C.

1977. Investigaciones Arqueológicas. Comp. Leila C. Tuya de Maeso. Imprenta Don Bosco. Montevideo.

Martínez, E., L. Cabrera, N. Fusco y C. Curbelo.

1989. Protección del patrimonio arqueológico: una propuesta integral. Boletín de Arqueología de la Comisión Nacional de Patrimonio 1: 15-20.

Ministerio de Educación y Cultura [MEC].

1977. Actas del V Encuentro de Arqueología del Litoral, noviembre de 1977. Fray Bentos. Ministerio de Educación y Cultura. Montevideo.

Ministerio de Educación y Cultura [MEC].

2017. Exposición arqueológica. "Arcilla, piedra y hueso a la orilla del Hum” en el marco de reapertura del Museo Maeso en la Villa Santo Domingo Soriano. Recuperado en 22 de abril de 2021 de https://www.gub.uy/ministerio-educacion- cultura/comunicacion/noticias/exposicionarqueologica-1

Museo de Historia del Arte.

s.f. Colecciones arqueológicas americanas. Intendencia de Montevideo. Recuperado en 22 de abril de 2021 de https://muhar.montevideo.gub.uy/coleccion0/colecciones-arqueologicas-americanas

Museo Nacional de Antropología [MNA].

s.f. a Institucional. Reseña Histórica. Ministerio de Educación y Cultura. Recuperado en 22 de abril de 2021 de https://www.mna.gub.uy/innovaportal/v/357 4/14/mecweb/resenahistorica $? 3$ colid $=3570 \&$ breadid $=$ null

Museo Nacional de Antropología [MNA].

s.f. b Violeta Bonino de Langguth. Ministerio de Educación y Cultura. Recuperado en 22 de abril de 2021 de https://www.mna.gub.uy/innovaportal/file/36 91/1/violeta-bonino.pdf

Museo Nacional de Antropología [MNA].

2019. Exposición "El acervo creció". Ministerio de Educación y Cultura. Recuperado en 22 de aril de 2021 de https://www.mna.gub.uy/mecweb/imprimir.j sp? contentid $=124166 \&$ site $=14 \&$ channel $=$ me cweb

Museo Nacional de Historia Natural [MNHN].

s.f. Biografías. Francisco Oliveras Acosta. Ministerio de Educación y Cultura. Recuperado en 22 de abril de 2021 de https://www.mnhn.gub.uy/innovaportal/v/36 96/12/mecweb/o---p--r?3 colid $=3085 \&$ breadid $=$ null

Museo Municipal de Historia Natural de Río Negro [MMHNRN].

1973. Antecedentes y Anales de los Congresos. Primer Congreso Nacional de Arqueología y Segundo Encuentro de Arqueología del Interior, 20-23 de diciembre de 1972. Museo Nacional de Historia Natural. Recuperado en 22 de abril de 2021 de https://www.mnhn.gub.uy/innovaportal/file/ 3717/1/pextra-20.pdf

Montón Subías, S.

2014. Arqueologías engeneradas. Breve introducción a los estudios de género en Arqueología hasta la actualidad. ArqueoWeb 15: 242-247. 
Navarrete, $R$.

2010. Excavando mujeres en y desde el sur: Aproximaciones a la arqueología feminista en Latinoamérica. Revista Venezolana de Estudios de la Mujer 15 (34): 75-104.

Pacheco, G.

2019. Relaciones de género: mujeres, sanmarquinas y chicheras. Desde el Sur 11 (2): 305-31.

Prados Torreira, L. y López Ruiz, C. (eds.).

2017. Museos arqueológicos y género. Educando en igualdad. UAM Ediciones. Madrid.

Rizzo, A.

1977. Consideraciones sobre el precerámico en el Sureste de la Provincia de Misiones, R.A. En $V$ Encuentro de Arqueología del Litoral: 211-244. MEC. Montevideo.

Rostagnol, S

1989. Relaciones de poder entre los géneros. En I Jornadas de Ciencias Antropológicas en el Uruguay: 193-197. Ministerio de Educación y Cultura. Montevideo.

ROU.

1996. Resolución $N^{\circ}$ 1019/996. Declaración de Monumento Histórico Nacional. Montevideo. Registro Nacional de Leyes y Decretos, Tomo 1, Semestre 2, Año 1996. Disponible en http://www.impo.com.uy/bases/resoluciones/ 1019-1996/1

Rouco, C., A. Díaz y J. Baeza.

1973. Métodos modernos aplicados a la cerámica del sitio arqueológico "Cerrito Vizcaíno" (Delta del Río Negro - Uruguay). En Primer Congreso Nacional de Arqueología del Uruguay, Segundo Encuentro de Arqueología del Litoral 6-16. CEA. Fray Bentos.

Sociedad de Amigos de la Arqueología [SAA].

1927. Revista de la Sociedad de Amigos de la Arqueología, Tomo I. Montevideo.

Sociedad de Amigos de la Arqueología [SAA].

1928. Revista de la Sociedad de Amigos de la Arqueología, Tomo II. Montevideo.

Sociedad de Amigos de la Arqueología [SAA].

1930. Revista de la Sociedad de Amigos de la Arqueología, Tomo IV. Montevideo.

Sociedad de Amigos de la Arqueología [SAA].

1931. Revista de la Sociedad de Amigos de la Arqueología, Tomo V. Montevideo.
Sociedad de Amigos de la Arqueología [SAA].

1932. Revista de la Sociedad de Amigos de la Arqueología, Tomo VI. Montevideo.

Sociedad de Amigos de la Arqueología [SAA].

1933. Revista de la Sociedad de Amigos de la Arqueología, Tomo VII. Montevideo.

Sociedad de Amigos de la Arqueología [SAA].

1947. Revista de la Sociedad de Amigos de la Arqueología, Tomo X. Montevideo.

Sociedad de Amigos de la Arqueología [SAA].

1956. Revista de la Sociedad de Amigos de la Arqueología, Tomo XIV. Montevideo.

Sociedad de Amigos de la Arqueología [SAA].

1957. Revista de la Sociedad de Amigos de la Arqueología, Tomo XV. Montevideo.

Sicardi, O. E.

1989 [1987]. Violeta Bonino de Langguth [obituario]. Comunicaciones de la Sociedad Malacológica del Uruguay 7 (52-53): 20.

Solnit, R.

2019. Los hombres me explican cosas. Fiordo. CABA.

Suárez, R.

2000. Tembetas, adornos, atlatls y otros instrumentos indígenas: La Colección Arqueológica del poeta Carlos Maeso Tognochi. Programa "Nuestro Pasado Indígena. Ministerio de Educación y Cultura. Montevideo.

Tavera Medina, $\mathrm{C}$.

2019. Una mirada feminista a la comunidad arqueológica peruana. Desde el Sur 11 (2): 239-260.

Toscano, A.

1989. Gestión del Museo Nacional de Antropología. $1^{a s}$ Jornadas de Ciencias Antropológicas en el Uruguay: 281-282. 23 al 27 de noviembre de 1987. Museo Nacional de Antropología. Montevideo.

Tuya de Maeso, L. C.

1973. Informe sobre el material cerámico estudiado por el arqueólogo Carlos Maeso. Conferencia dada en el Primer Congreso Nacional de Arqueología y Segundo Encuentro de Arqueología del Interior, 22 de diciembre de 1972. CEA. Fray Bentos.

Tuya de Maeso, L. C.

1980. Consideraciones sobre la cerámica de yacimientos arqueológicos investigados en 
los departamentos de Colonia, Soriano y Río Negro: decoración, expresiones zoomorfas. Imprenta Don Bosco. Montevideo.

Voss, B.

2021 a. Documenting cultures of harassment in Archaeology: A review and analysis of quantitative and qualitative research studies. American Antiquity $86 \quad$ (2): 1-17. https://doi.org/10.1017/aaq.2020.118

Voss, B.

2021 b. Disrupting cultures of harassment in Archaeology: Social-environmental and trauma-informed approaches to disciplinary transformation. American Antiquity 86 (3): 1-18. https://doi.org/10.1017/aaq.2021.19

Wolf, V.

[1929] 2008. Una habitación propia. Editorial Seix Barral. Barcelona.

Wylie, A.

1997. The engendering of Archaeology. Refiguring feminist science studies. Osiris12 Women, Gender, and Science: New Directions: 8099. The University of Chicago Press. http://www.jstor.org/stable/301900

\footnotetext{
${ }^{1}$ Coordinador del Área de Gestión Territorial del Dpto. de Cultura de la Intendencia de Soriano, Uruguay

${ }^{2}$ La revista "El Grillo" fue una publicación periódica con una tirada de 100.000 ejemplares que dio inicio en el año 1949 por iniciativa del Cuerpo Legislativo del País orientada al público escolar de Uruguay, y cuyo último número fue publicado en el año 1966 (Brymer y Pontet, 2006).

${ }^{3}$ En el artículo de Brymer y Pontet (2006), así como en las notas de prensa (https://brecha.com.uy/el-uruguayilustrado/ - https://ladiaria.com.uy/articulo/2014/2/pequenos-artistas/) se ha rescatado la historia tanto de Bell Clavelli como de Mercedes Antello. Pero estas son todas publicaciones realizadas por mujeres ajenas a la arqueología y que no tratan de ese vínculo en particular de su trabajo.
}

${ }^{4}$ La propuesta curatorial está a cargo del MNA, dirigido actualmente por la Mag. Carina Erchini, y cuenta con la participación de investigadoras externas, entre quienes nos encontramos las autoras de este trabajo. https://www.mna.gub.uy/innovaportal/v/125259/14/mecweb/exposicion:-miradas-pioneras-de-la-arqueologiauruguaya?parentid=123586

${ }^{5}$ La exposición fue inaugurada el 12 de julio de 2021.

${ }^{6}$ En masculino en el original.

${ }^{7}$ Queremos destacar que hay una investigación en curso de la Directora del MNA Carina Erchini sobre la figura de Violeta Bonino y sus aportes a la arqueología. Por este motivo, lo que se presenta aquí es muy breve y de carácter absolutamente preliminar.

${ }^{8}$ Este no puede considerarse un número cerrado ya que a medida que avanza la investigación se van recuperando nombres de más mujeres que fueron socias del CEA o que participaron de alguna manera en sus actividades.

${ }^{9}$ Antonia Rizzo (1929-2008) fue Doctora en Historia y docente de Antropología de las facultades de Ciencias Naturales y Museo de la Universidad Nacional de La Plata, de Filosofía y Letras de la Universidad de Buenos Aires, y de la Universidad de El Salvador. Más datos sobre la autora: http://revistascientificas.filo.uba.ar/index.php/Arqueologia/article/view/1714

${ }^{10}$ Por detalles sobre vida y obra de Annette Laming-Emperaire se puede consultar Lavallée (1978).

${ }^{11}$ Queda por estudiar aún también la participación de estas mujeres en los congresos regionales como los Nacionales de Arqueología Argentina y los de la Sociedad de Arqueología Brasilera. 